\title{
ON THE FOURTH COEFFICIENT OF BOUNDED UNIVALENT FUNCTIONS
}

\author{
BY \\ M. SCHIFFER AND O. TAMMI(1)
}

1. Let $S\left(b_{1}\right)$ be the class of univalent bounded functions in the unit disc with the series development

$$
\begin{aligned}
f(z) & =\sum_{\nu=1}^{\infty} b_{v} z^{\nu}, \quad 0<b_{1} \leqq 1, \\
|f(z)| & \leqq 1, \quad|z| \leqq 1
\end{aligned}
$$

This class has often been studied in the equivalent form of normalized univalent functions

$$
\begin{gathered}
F(z)=b_{1}^{-1} f(z)=z+\sum_{v=2}^{\infty} a_{v} z^{v} \\
a_{v}=\frac{b_{v}}{b_{1}}, \quad|F(z)| \leqq b_{1}^{-1}
\end{gathered}
$$

We shall study estimates for the coefficient $a_{4}$ in dependence on the first coefficient $b_{1} . \mathrm{J}$ For this purpose an inequality of the Grunsky-Nehari type [2], [3] will be used. Such inequalities were first applied by V. Singh [4] in studying the coefficient $a_{4}$ of bounded univalent functions with real coefficients. The method of Singh, which follows the argument of Charzynski and Schiffer [1] in the case of unbounded univalent functions, can be extended to the case of nonreal coefficients. Though much harder to apply here than in the real case, this method yields sharp bounds for $a_{4}$ in an interval of $b_{1}$-values near $b_{1}=0$ and in an interval near $b_{1}=1$.

2. We consider the univalent and bounded function

$$
F(z)=\sqrt{ } f\left(z^{2}\right)
$$

and apply to it the Grunsky-Nehari inequalities for such functions. We use specifically the estimate

$$
\begin{aligned}
& \left|a_{4}-2 a_{2} a_{3}+\frac{13}{12} a_{2}^{3}+x_{1}^{2} a_{2}+x_{2}^{2}\left(a_{3}-a_{2}^{2}\right)+2 x_{1}\left(a_{3}-\frac{3}{4} a_{2}^{2}\right)\right| \\
& \leqq \frac{2}{3}\left(1-b_{1}^{3}\right)-\frac{1}{2} b_{1}\left|a_{2}\right|^{2}+2\left|x_{1}\right|^{2}\left(1-b_{1}\right)+\left|x_{2}\right|^{2}\left(1-b_{1}^{2}\right)-2 b_{1} \operatorname{Re}\left\{x_{1} \bar{a}_{2}\right\}
\end{aligned}
$$

Received by the editors September 8, 1964.

(1) This work was suuported in part by AF 49(638)1345, Stanford University. 
Here $x_{1}$ and $x_{2}$ are arbitrary complex parameters. We simplify this inequality by selecting

$$
x_{2}=0, \quad x_{1}=l=\text { real }
$$

We find

$$
\left|a_{4}-2 a_{2} a_{3}+\frac{13}{12} a_{2}^{3}+2\left(a_{3}-\frac{3}{4} a_{2}^{2}\right) l+a_{2} l^{2}\right|
$$

$$
\leqq \frac{2}{3}\left(1-b_{1}^{3}\right)-\frac{1}{2} b_{1}\left|a_{2}\right|^{2}+2 l^{2}\left(1-b_{1}\right)-2 l b_{1} \operatorname{Re}\left\{a_{2}\right\}
$$

This is the same estimate which Singh used in the case of real coefficients [4]. There is no loss of generality if we assume

$$
a_{4}>0, \quad \operatorname{Re}\left\{a_{2}\right\} \geqq 0,
$$

since we can always attain this situation for any function of the class $S\left(b_{1}\right)$ by a trivial rotation. In this case (6) can be weakened to

$$
a_{4} \leqq \frac{2}{3}\left(1-b_{1}^{3}\right)+2 \operatorname{Re}\left\{a_{2} \lambda\right\}+\frac{5}{12} \operatorname{Re}\left\{a_{2}^{3}\right\}-\frac{1}{2} b_{1}\left|a_{2}\right|^{2}
$$

$$
-2 l\left(\operatorname{Re}\{\lambda\}+b_{1} \operatorname{Re}\left\{a_{2}\right\}\right)+l^{2}\left(2\left(1-b_{1}\right)-\operatorname{Re}\left\{a_{2}\right\}\right)
$$

with

$$
\lambda=a_{3}-\frac{3}{4} a_{2}^{2}
$$

The inequality (8) implies, in particular,

$$
2\left(1-b_{1}\right)-\operatorname{Re}\left\{a_{2}\right\} \geqq 0 .
$$

It is well known that equality in this estimate is possible only for the function

$$
f(z)=p^{-1}\left[b_{1} p(z)\right], \quad p(z)=\frac{z}{(1-z)^{2}},
$$

which maps $|z|<1$ onto the unit disc minus a radial slit along the negative axis. Assuming for the moment that we are not dealing with this particular function, we can minimize the right-hand side of (8) by choosing

$$
l=\frac{\operatorname{Re}\{\lambda\}+b_{1} \operatorname{Re}\left\{a_{2}\right\}}{2\left(1-b_{1}\right)-\operatorname{Re}\left\{a_{2}\right\}}
$$

and find the most favorable estimate 


$$
\begin{aligned}
a_{4} \leqq & \frac{2}{3}\left(1-b_{1}^{3}\right)+2 \operatorname{Re}\left\{a_{2} \lambda\right\}+\frac{5}{12} \operatorname{Re}\left\{a_{2}^{3}\right\}-\frac{1}{2} b_{1}\left|a_{2}\right|^{2} \\
& -\frac{\left(\operatorname{Re}\{\lambda\}+b_{1} \operatorname{Re}\left\{a_{2}\right\}\right)^{2}}{2\left(1-b_{1}\right)-\operatorname{Re}\left\{a_{2}\right\}}=Y .
\end{aligned}
$$

In the case of the exceptional function (10) the coefficients of $l$ and $l^{2}$ in (8) vanish and $a_{4}$ equals the remaining right-hand terms. An easy calculation yields in this case

$$
a_{4}=4-20 b_{1}+30 b_{1}^{2}-14 b_{1}^{3} .
$$

We return to the general case of the inequality (12) and rearrange its right-hand side $Y$ as follows:

$$
Y=\frac{2}{3}\left(1-b_{1}^{3}\right)-\frac{5}{2} b_{1}\left(\operatorname{Re}\left\{a_{2}\right\}\right)^{2}+\frac{5}{12} \operatorname{Re}\left\{a_{2}^{3}\right\}-2 \operatorname{Im}\left\{a_{2}\right\} \operatorname{Im}\{\lambda\}
$$

$$
-\frac{1}{2} b_{1}\left(\operatorname{Im}\left\{a_{2}\right\}\right)^{2}+2 \operatorname{Re}\left\{a_{2}\right\}\left[\operatorname{Re}\{\lambda\}+b_{1} \operatorname{Re}\left\{a_{2}\right\}\right]-\frac{\left(\operatorname{Re}\{\lambda\}+b_{1} \operatorname{Re}\left\{a_{2}\right\}\right)^{2}}{2\left(1-b_{1}\right)-\operatorname{Re}\left\{a_{2}\right\}}
$$

The reason for this rearrangement is that we wish to replace everywhere $\operatorname{Re}\{\lambda\}$ by the combination $\operatorname{Re}\{\lambda\}+b_{1} \operatorname{Re}\left\{a_{2}\right\}$ and to replace the right-hand side of $Y$ by the largest value obtainable for any choice of this combination. We easily find

$$
\begin{aligned}
Y \leqq & \frac{2}{3}\left(1-b_{1}^{3}\right)-\frac{5}{2} b_{1}\left(\operatorname{Re}\left\{a_{2}\right\}\right)^{2}+\frac{5}{12} \operatorname{Re}\left\{a_{2}^{3}\right\}-2 \operatorname{Im}\left\{a_{2}\right\} \operatorname{Im}\{\lambda\} \\
& -\frac{1}{2} b_{1}\left(\operatorname{Im}\left\{a_{2}\right\}\right)^{2}+\left(\operatorname{Re}\left\{a_{2}\right\}\right)^{2}\left(2\left(1-b_{1}\right)-\operatorname{Re}\left\{a_{2}\right\}\right)
\end{aligned}
$$

and by a final rearrangement arrive at the following estimate which holds for all functions in $S\left(b_{1}\right)$ except for the function (10):

$$
a_{4} \leqq \frac{2}{3}\left(1-b_{1}^{3}\right)+\frac{1}{2}\left(4-9 b_{1}\right)\left(\operatorname{Re}\left\{a_{2}\right\}\right)^{2}-\frac{7}{12}\left(\operatorname{Re}\left\{a_{2}\right\}\right)^{3}
$$

$$
-\frac{5}{4} \operatorname{Re}\left\{a_{2}\right\}\left(\operatorname{Im}\left\{a_{2}\right\}\right)^{2}-2 \operatorname{Im}\left\{a_{2}\right\} \operatorname{Im}\{\lambda\}-\frac{1}{2} b_{1}\left(\operatorname{Im}\left\{a_{2}\right\}\right)^{2}
$$

This is the fundamental inequality which will be the basis for our further estimates. We note again the definition

$$
\lambda=a_{3}-\frac{3}{4} a_{2}^{2}
$$

of the auxiliary parameter. 
3. One important problem in the further evaluation of (16) is the removal of the parameter $\lambda$ by means of an additional inequality. In the case of unbounded univalent functions the elimination of $\lambda$ was based upon the estimate

$$
3|\lambda|^{2} \leqq 4-\left|a_{2}\right|^{2}
$$

which is an immediate consequence of the area principle [1]. In the present case of bounded functions we have to derive an analogous but more specific estimate.

We construct the function

$$
G(z)=\frac{1}{\sqrt{ } f\left(z^{2}\right)}-\sqrt{ } f\left(z^{2}\right)=\frac{1}{\sqrt{ } b_{1}}\left(\frac{1}{z}+\alpha_{1} z+\alpha_{3} z^{3}+\cdots\right)
$$

which is likewise univalent; and since $\sqrt{ } f\left(z^{2}\right)$ is bounded, we can assert that $G(z)$ maps $|z|<1$ into the complex plane slit along the imaginary axis from $-2 i$ to $2 i$. Using the power-series developments

$$
\begin{aligned}
& \sqrt{ } f\left(z^{2}\right)=\frac{1}{\sqrt{ } b_{1}}\left(b_{1} z+\frac{1}{2} b_{1} a_{2} z^{3}+\cdots\right), \\
& \frac{1}{\sqrt{ } f\left(z^{2}\right)}=\frac{1}{\sqrt{ } b_{1}}\left(\frac{1}{z}-\frac{1}{2} a_{2} z-\frac{1}{2} \lambda z^{3}+\cdots\right)
\end{aligned}
$$

we find

$$
G(z)=\frac{1}{\sqrt{ } b_{1}}\left[\frac{1}{z}-\left(b_{1}+\frac{1}{2} a_{2}\right) z-\frac{1}{2}\left(b_{1} a_{2}+\lambda\right) z^{3}+\cdots\right] .
$$

Since $\sqrt{ } b_{1} G(z)$ is a normalized univalent function in $|z|<1$, we can conclude from the classical area theorem that

$$
\left|b_{1}+\frac{1}{2} a_{2}\right|^{2}+\frac{3}{4}\left|b_{1} a_{2}+\lambda\right|^{2}+\cdots \leqq 1 .
$$

Hence we have the estimate

$$
\frac{3}{4}\left|b_{2}+\lambda\right|^{2} \leqq 1-\left|b_{1}+\frac{a_{2}}{2}\right|^{2} .
$$

This inequality is sharp in the case of the function (10) as can easily be verified.

Because of the inequality (21) it is convenient to replace the parameter $\lambda$ by the new auxiliary quantity

$$
\mu=\lambda+b_{2}
$$

and then express (21) in the form

$$
3|\mu|^{2} \leqq 4-\left|2 b_{1}+a_{2}\right|^{2} .
$$


We further introduce the quantities

$$
\begin{aligned}
& \operatorname{Re}\left\{a_{2}\right\}=2\left(1-b_{1}\right)-x, \quad 0<x \leqq 2\left(1-b_{1}\right), \\
& \operatorname{Im}\left\{a_{2}\right\}=y, \quad \operatorname{Re}\{\mu\}=\xi, \quad \operatorname{Im}\{\mu\}=\eta .
\end{aligned}
$$

With these notations we may bring the inequality (16) into the form

$$
\begin{aligned}
a_{4} \leqq & \frac{2}{3}\left(1-b_{1}^{3}\right)+\frac{1}{2}\left(4-9 b_{1}\right)\left[2\left(1-b_{1}\right)-x\right]^{2}-\frac{7}{12}\left[2\left(1-b_{1}\right)-x\right]^{3} \\
& -\frac{5}{4}\left[2\left(1-b_{1}\right)-x\right] y^{2}-2 y \eta+\frac{3}{2} b_{1} y^{2} \\
= & 4-20 b_{1}+30 b_{1}^{2}-14 b_{1}^{3}-\left(1-b_{1}\right)\left(1-11 b_{1}\right) x-\frac{1}{2}\left(3+2 b_{1}\right) x^{2} \\
& +\frac{7}{12} x^{3}-\left(\frac{5}{2}-4 b_{1}-\frac{5}{4} x\right) y^{2}-2 y \eta .
\end{aligned}
$$

Following the procedure in [1], we write

$$
-2 y \eta=\alpha y^{2}+\frac{1}{\alpha} \eta^{2}-\alpha\left(y+\frac{1}{\alpha} \eta\right)^{2}
$$

with a free parameter $\alpha>0$ and transform (24) into the inequality

$$
a_{4}-\left(4-20 b_{1}+30 b_{1}^{2}-14 b_{1}^{3}\right) \leqq\left(1-b_{1}\right)\left(11 b_{1}-1\right) x-\frac{1}{2}\left(3+2 b_{1}\right) x^{2}
$$

$$
+\frac{7}{12} x^{3}-\left(\frac{5}{2}-4 b_{1}-\frac{5}{4} x\right) y^{2}+\alpha y^{2}+\frac{1}{\alpha} \eta^{2} .
$$

In view of $\left(21^{\prime}\right)$ we have

$$
3\left(\xi^{2}+\eta^{2}\right) \leqq 4 x-x^{2}-y^{2},
$$

i.e.,

$$
\eta^{2} \leqq \frac{4}{3} x-\frac{1}{3}\left(x^{2}+y^{2}\right)
$$

By means of this estimate we can now eliminate $\eta$ from (26) and arrive at

$$
a_{4}-\left(4-20 b_{1}+30 b_{1}^{2}-14 b_{1}^{3}\right) \leqq\left[\frac{4}{3 \alpha}+\left(1-b_{1}\right)\left(11 b_{1}-1\right)\right] x
$$

$$
-\left(\frac{3+2 b_{1}}{2}+\frac{1}{3 \alpha}\right) x^{2}+\frac{7}{12} x^{3}-\left(\frac{5}{2}-4 b_{1}+\frac{1}{3 \alpha}-\alpha-\frac{5}{4} x\right) y^{2} .
$$

As in [1], it will be convenient to apply the inequality (28) with two different choices of $\alpha>0$. We start with 
Case I.

$$
\frac{4}{3 \alpha}=\left(1-b_{1}\right)\left(1-11 b_{1}\right), \quad \alpha=\frac{4}{3\left(1-b_{1}\right)\left(1-11 b_{1}\right)}>0
$$

which is admissible for $b_{1}<1 / 11$.

This assumption yields the estimate

$$
a_{4}-\left(4-20 b_{1}+30 b_{1}^{2}-14 b_{1}^{3}\right) \leqq-A x^{2}-B y^{2}
$$

with

$$
A=\frac{1}{4}\left[7-8 b_{1}+11 b_{1}^{2}-\frac{7}{3} x\right],
$$

$$
B=\frac{5}{2}-4 b_{1}+\frac{\left(1-b_{1}\right)\left(1-11 b_{1}\right)}{4}-\frac{4}{3} \frac{1}{\left(1-b_{1}\right)\left(1-11 b_{1}\right)}-\frac{5}{4} x
$$

We wish to prove that the left side in (30) is nonpositive, for $b_{1}$ sufficiently small. For this purpose it is enough to determine bounds for $b_{1}$ which guarantee that $A$ and $B$ are non-negative. We start with the condition

$$
A \geqq 0, \quad \text { i.e., } x \leqq \frac{3}{7}\left(7-8 b_{1}+11 b_{1}^{2}\right) .
$$

Since we know from (7) the inequality $x \leqq 2\left(1-b_{1}\right)$, we are led to the sufficient condition

$$
2\left(1-b_{1}\right) \leqq \frac{3}{7}\left(7-8 b_{1}+11 b_{1}^{2}\right)
$$

that is,

$$
33 b_{1}^{2}-10 b_{1}+7>0 \text {. }
$$

But this inequality is true for all real values $b_{1}$. Hence $A>0$ for all values $0 \leqq x \leqq 2\left(1-b_{1}\right)$.

Next we discuss the conditions which guarantee $B \geqq 0$. That is,

$$
x \leqq \frac{1}{5}\left[11-28 b_{1}+11 b_{1}^{2}-\frac{16}{3} \frac{1}{\left(1-b_{1}\right)\left(1-11 b_{1}\right)}\right]=\phi\left(b_{1}\right) .
$$

We easily verify that $\phi\left(b_{1}\right)$ decreases monotonically as $b_{1}$ varies from zero to the value $1 / 11$. We can assert that

$$
a_{4}<4-20 b_{1}+30 b_{1}^{2}-14 b_{1}^{3}
$$

as long as $0<x \leqq \phi\left(b_{1}\right)$. To discuss the validity of (34) for values $x>\phi\left(b_{1}\right)$, we change now the value of $\alpha$ in the inequality (28) and proceed to 
Case II.

$$
\alpha=\frac{1}{2}:
$$

In this case we may express (28) in the form

$$
a_{4}-\left(4-20 b_{1}+30 b_{1}^{2}-14 b_{1}^{3}\right) \leqq-C x-D y^{2}
$$

with

$$
\begin{aligned}
& C=-\frac{1}{3}\left[\left(5+36 b_{1}-33 b_{1}^{2}\right)-\frac{1}{2}\left(13+6 b_{1}\right) x+\frac{7}{4} x^{2}\right], \\
& D=\frac{8}{3}-4 b_{1}-\frac{5}{4} x .
\end{aligned}
$$

Clearly, by the bounds on $x$ in (23) we have

$$
D>\frac{8}{3}-4 b_{1}-\frac{5}{2}\left(1-b_{1}\right)=\frac{1}{6}-\frac{3}{2} b_{1}
$$

and this quantity is non-negative for

$$
b_{1}<\frac{1}{9}
$$

Since $x>0$, the validity of the inequality (34) depends then only on the sign of the polynomial

$$
r(x)=5+36 b_{1}-33 b_{1}^{2}-\frac{1}{2}\left(13+6 b_{1}\right) x+\frac{7}{4} x^{2} .
$$

Since (34) is verified for $x \leqq \phi\left(b_{1}\right)$, we have only to show that $r(x)$ is negative for values

$$
\phi\left(b_{1}\right) \leqq x \leqq 2\left(1-b_{1}\right), \quad 0<b_{1}<\frac{1}{9}
$$

Since $r(x)$ is a polynomial of second degree which becomes large positive for large values of $x$, it is sufficient to guarantee that $r(x)$ is negative at the end points of the interval (40) in order to ensure its negative character inside. We easily calculate

$$
r\left[2\left(1-b_{1}\right)\right]=-1+29 b_{1}-20 b_{1}^{2}<0 \text { for } 0 \leqq b_{1}<\frac{1}{29} .
$$

A somewhat more complicated numerical test shows that

$$
r\left[\phi\left(b_{1}\right)\right]<0 \text { for } 0 \leqq b_{1} \leqq \frac{1}{700} 1
$$

We thus proved: 
THEOREM I. In the class $S\left(b_{1}\right)$ of bounded univalent functions the fourth coefficient satisfies the inequality

$$
\left|a_{4}\right| \leqq 4-20 b_{1}+30 b_{1}^{2}-14 b_{1}^{3}
$$

at least in the interval

$$
0 \leqq b_{1} \leqq \frac{1}{700} .
$$

Equality in (43) is possible only in the case of the radial slit mapping (10).

4. We have used rather crude estimates to obtain the result summarized in Theorem I. The main intention until now was to show that the inequality (4) leads to an estimate for the fourth coefficient of a univalent bounded function for small enough $b_{1}$, even if the assumption on the reality of the coefficients is dropped. In this section we shall now try to improve the range of $b_{1}$ for which the estimate (43) is valid by optimal choice of the parameter $\alpha$ in the inequality (28).

Indeed, we may select for given values of $x$ and $y$ the parameter $\alpha$ such that the right-hand side of (28) becomes minimal. We rearrange (28) in the form

$$
\begin{aligned}
a_{4}-\left(4-20 b_{1}+30 b_{1}^{2}-14 b_{1}^{3}\right) \leqq \frac{x}{3} & {\left[3\left(1-b_{1}\right)\left(11 b_{1}-1\right)-\frac{3}{2}\left(3+2 b_{1}\right) x+\frac{7}{4} x^{2}\right] } \\
(45) & -\left(\frac{5}{2}-4 b_{1}-\frac{5}{4} x\right) y^{2}+\frac{4 x-x^{2}-y^{2}}{3 \alpha}+y^{2} \alpha .
\end{aligned}
$$

Clearly

$$
\frac{4 x-x^{2}-y^{2}}{3 \alpha}+y^{2} \alpha \geqq 2|y| \sqrt{\frac{4 x-x^{2}-y^{2}}{3}}
$$

for all positive $\alpha$, provided that $4 x-x^{2}-y^{2} \geqq 0$. This inequality must hold since the right-hand side in (45) is bounded from below for all positive values of $\alpha$. If we use the definitions (23) of $x$ and $y$, we find that this condition reduces to

$$
\left|2 b_{1}+a_{2}\right|^{2} \leqq 4
$$

which is an immediate and trivial consequence of the area theorem (20).

To obtain equality in (46), we have to choose

$$
\alpha=\sqrt{\frac{4 x-x^{2}-y^{2}}{3 y^{2}}>0 .}
$$

The case $y=0$ may be considered as a limit case and does not give any special difficulty. We can always assert the inequality

$$
\begin{aligned}
a_{4}-\left(4-20 b_{1}+30 b_{1}^{2}-14 b_{1}^{3}\right) \leqq & \frac{x}{3} t(x)-\left(\frac{5}{2}-4 b_{1}-\frac{5}{4} x\right) y^{2} \\
& +2|y| \sqrt{\frac{4 x-x^{2}-y^{2}}{3}}=F(x, y) .
\end{aligned}
$$


Here $t(x)$ is the quadratic polynomial in $x$ :

$$
t(x)=3\left(1-b_{1}\right)\left(11 b_{1}-1\right)-\frac{3}{2}\left(3+2 b_{1}\right) x+\frac{7}{4} x^{2} .
$$

Next we will ask for the maximum value of the right-hand term $F(x, y)$ for fixed $x$ and all admissible values $y$. Since $F(x, y)$ is even in $y$, we may assume without loss of generality that $y \geqq 0$.

We use the abbreviations

$$
U=\frac{5}{2}-4 b_{1}-\frac{5}{4} x, \quad V=\frac{1}{3}\left(4 x-x^{2}\right)
$$

and consider the equivalent problem to maximize the function

$$
q(y)=2 y \sqrt{ }\left(V-\frac{1}{3} y^{2}\right)-U y^{2}
$$

in the interval $0 \leqq y \leqq \sqrt{ }(3 V)$. Straightforward differentiation leads to the following condition for the value of $y$ at interval extrema:

$$
\frac{V-\frac{2}{3} y^{2}}{\sqrt{ }\left(V-\frac{1}{3} y^{2}\right)}=U y .
$$

Solving this algebraic equation for $y$, we obtain the determinations for the possible extremum points:

$$
\begin{aligned}
& y_{1}^{2}=\frac{3}{2} V\left[1-\sqrt{\frac{3 U^{2}}{4+U^{2}}}\right], \\
& y_{2}^{2}=\frac{3}{2} V\left[1+\sqrt{\frac{3 U^{2}}{4+U^{2}}}\right] .
\end{aligned}
$$

However, it should be observed that in the case $U>0$, equation (53) requires $V-2 y^{2} / 3>0$ if we look for positive $y$-values. In this case only $y_{1}^{2}$ will be permissible. On the other hand, for $U<0$ we have to demand $V-2 y^{2} / 3<0$, which leads to the only possibility $y_{2}^{2}$. Thus we found: The internal extremal point for $q(y)$ satisfies the equation

$$
y^{2}=\frac{3}{2} V\left[1-\frac{U}{\sqrt{\left(\frac{4}{3}+U^{2}\right)}}\right] .
$$

It is easily seen that $q^{\prime}(y)$ is positive at $y=0$ and tends to negative infinity values at $y=\sqrt{ }(3 V)$. Thus the point (55) leads to a maximum of $q(y)$, and we find from (52) and (55) 


$$
\max q(y)=-\frac{3}{2} U V+\frac{3}{2} V \sqrt{ }\left(\frac{3}{4}+U^{2}\right) .
$$

We insert this maximum value of $q(y)$ into the right side of (49) and obtain the result:

THEOREM II. Using the best possible choice of the positive parameter $\alpha$, we obtain the following estimate for $a_{4}$ in terms of $x$ :

$$
a_{4}-\left(4-20 b_{1}+30 b_{1}^{2}-14 b_{1}^{3}\right) \leqq \frac{1}{3} x M(x)
$$

with

$$
\begin{aligned}
& M(x)=3\left(1-b_{1}\right)\left(11 b_{1}-1\right)-\frac{3}{2}\left(3+2 b_{1}\right) x+\frac{7}{4} x^{2} \\
& +\frac{3}{2}(4-x)\left[\sqrt{ }\left(\frac{4}{3}+\left(\frac{5}{2}-4 b_{1}-\frac{5}{4} x\right)^{2}\right)-\left(\frac{5}{2}-4 b_{1}-\frac{5}{4} x\right)\right] .
\end{aligned}
$$

By use of this inequality we can improve the interval for $b_{1}$, for which the estimate (43) is valid. Indeed, this inequality depends solely on the sign of $M(x)$ in the interval $0<x \leqq 2\left(1-b_{1}\right)$.

We are indebted to Dr. G. Forsythe for evaluating $M(x)$ on a digital computer in the intervals needed for various values of $b_{1}$. It was found that $M(x)$ is negative at least for all values of $b_{1} \leqq 3 / 100$ and the estimate (43) has thus been established up to this value of $b_{1}$. It was also found that $M(0)$ is positive for $b_{1}=1 / 25$, which shows that our method cannot be extended beyond this point.

5. We turn now to the problem of estimating the coefficient $a_{4}$, as the first coefficient $b_{1}$ is near its other extreme value, namely 1 . For this purpose we start again with our initial inequality (4) and choose

$$
x_{1}=a_{2}, \quad x_{2}=0
$$

which yields

$$
\left|a_{4}+\frac{7}{12} a_{2}^{3}\right| \leqq \frac{2}{3}\left(1-b_{1}^{3}\right)+\frac{1}{2}\left|a_{2}\right|^{2}\left(4-9 b_{1}\right) .
$$

This implies

$$
\operatorname{Re}\left\{a_{4}\right\} \leqq \frac{2}{3}\left(1-b_{1}^{3}\right)+\frac{1}{2}\left|a_{2}\right|^{2}\left(4-9 b_{1}\right)-\frac{7}{12} \operatorname{Re}\left\{a_{2}^{3}\right\} .
$$

Instead of (7) we make now the assumption

$$
a_{4}^{\prime}>0, \quad a_{2} !=: u 1+i v, \quad u \leqq 0 .
$$


This normalization can always be achieved by a properly chosen rotation. We bring (61) into the form

$$
a_{4} \leqq \frac{2}{3}\left(1-b_{1}^{3}\right)+\frac{1}{2}\left(4-9 b_{1}\right) u^{2}-\frac{7}{12} u^{3}+v^{2}\left[\frac{1}{2}\left(4-9 b_{1}\right)+\frac{7}{4} u\right]
$$

Using the estimate

$$
0 \geqq u \geqq-2\left(1-b_{1}\right)
$$

we find

$$
\frac{1}{2}\left(4-9 b_{1}\right)+\frac{7}{2} u \leqq 0 \quad \text { for } b_{1} \geqq \frac{4}{9}
$$

and

$$
u^{2}\left[\frac{1}{2}\left(4-9 b_{1}\right)-\frac{7}{12} u\right] \leqq \frac{1}{6} u^{2}\left[19-34 b_{1}\right] \leqq 0 \quad \text { for } b_{1} \geqq \frac{19}{34}
$$

Hence we proved

$$
a_{4} \leqq \frac{2}{3}\left(1-b_{1}^{3}\right) \quad \text { for } 1 \geqq b_{1} \geqq \frac{19}{34}
$$

There arises finally the question of the extremum function for which equality holds in (67). Observe that the additional terms in (63) can only vanish for $b_{1}$ $>19 / 34$ if $a_{2}=u+i v=0$. Hence we must seek the extremum function in the subclass of $S\left(b_{1}\right)$ with $a_{2}=0$. For such functions we can write (4) in the form $\left(x_{2}=0\right)$

$$
\left|a_{4}+2 x_{1} a_{3}\right| \leqq \frac{2}{3}\left(1-b_{1}^{3}\right)+2\left|x_{1}\right|^{2}\left(1-b_{1}\right)
$$

Choose now $x_{1}=\bar{a}_{3}$ and assume $a_{4}>0$; we obtain

$$
a_{4} \leqq \frac{2}{3}\left(1-b_{1}^{3}\right)-2 b_{1}\left|a_{3}\right|^{2}
$$

Thus equality in (67) is possible only for functions in $S\left(b_{1}\right)$ with $a_{2}=a_{3}=0$.

Finally we could characterize the extremum function for the inequality (67) within $S\left(b_{1}\right)$ by the variational differential equation. Using the Marty relations which follow from the differential equation, we can express $b_{4}, b_{5}, \cdots$ as functions with real coefficients in terms of $b_{1}, b_{2}$ and $b_{3}$. Hence it is evident that the extremum function must have real coefficients and maps the disc onto a domain symmetric with respect to the real axis.

Singh [4] has studied the special case of real functions in $S\left(b_{1}\right)$ and has determined the extremum function for (67) in this subclass. We have now shown that no additional extremum function can exist. 


\section{REFERENCES}

1. Z. Charzynski and M. Schiffer, A new proof of the Bieberbach conjecture for the fourth coefficient, Arch. Rational Mech. Anal. 5 (1960), 187-193.

2. H. Grunsky, Koeffizientenbedingungen für schlicht abbildende meromorphe Funktionen, Math. Z. 45 (1939), $29-61$.

3. Z. Nehari, Some inequalities in the theory of functions, Trans. Amer. Math. Soc. 75 (1953), 256-286.

4. V. Singh, Grunsky inequalities and coefficients of bounded schlicht functions, Ann. Acad. Sci. Fenn. Ser. AI No. 310 (1962).

\section{STANFORD UNIVERSITY,}

Stanford, CaLIFornia 